\title{
ON A RECURRENCE FOR PERMANENTS OF A SEQUENCE OF 3-TRIDIAGONAL MATRICES
}

\author{
Pavel Trojovský, Iva Zvoníková \\ Department of Mathematics, Faculty of Science \\ University of Hradec Králové, Hradec Králové, Czech Republic \\ pavel.trojovsky@uhk.cz,ivazvonikova@gmail.com
}

Received: 14 July 2019; Accepted: 11 December 2019

\begin{abstract}
This is a corrigendum of the paper: Küçük, A. Z. \& Düz, M. (2017). Relationships between the permanents of a certain type of $k$-tridiagonal symmetric Toeplitz and the Chebyshev polynomials. Journal of Applied Mathematics and Computational Mechanics, 16, 75-86. We will show that Remark 9, on page 84, does not hold, what is the consequence of the incorrect proof, which authors formulated there.
\end{abstract}

MSC 2010: 15A15, $11 B 05$

Keywords: permanent, $k$-tridiagonal matrix, Toeplitz matrix, recurrence relation, Chebyshev polynomial of the second kind

\section{Introduction}

The so-called $k$-tridiagonal matrices (this name was introduced by El-Mikkawy and Sogabe [1]) were first studied by Egerváry and Szász in [2]. Perhaps the most important non-trivial case is due to Losonczi [3]. A very recent and important survey in this topic can be found in da Fonseca and Kowalenko [4].

The $k$-tridiagonal matrices $\mathbf{T}_{n}^{(k)}\left(\mathbf{D}_{-k}, \mathbf{D}_{0}, \mathbf{D}_{k}\right)$ are defined by the following way

$$
\left(\begin{array}{ccccccccc}
d_{1} & 0 & \cdots & \cdots & 0 & a_{1} & 0 & \cdots & 0 \\
0 & d_{2} & \ddots & \ddots & \ddots & 0 & \ddots & \ddots & \vdots \\
\vdots & \ddots & \ddots & \ddots & \ddots & \ddots & \ddots & \ddots & 0 \\
0 & \ddots & \ddots & \ddots & 0 & \ddots & \ddots & \ddots & a_{n-k} \\
0 & \ddots & \ddots & \ddots & d_{k} & 0 & \ddots & \ddots & 0 \\
b_{k+1} & \ddots & \ddots & \ddots & 0 & d_{k+1} & \ddots & \ddots & 0 \\
0 & \ddots & \ddots & \ddots & \ddots & \ddots & \ddots & \ddots & \vdots \\
\vdots & \ddots & \ddots & \ddots & \vdots & \vdots & \ddots & \ddots & 0 \\
0 & \cdots & 0 & b_{n} & 0 & \cdots & \cdots & 0 & d_{n}
\end{array}\right)_{n \times n}
$$


where sequences $\left\{d_{j}\right\}_{j=1}^{n},\left\{a_{j}\right\}_{j=1}^{n-k}$ and $\left\{b_{j}\right\}_{j=k+1}^{n}$ create the main diagonal $\mathbf{D}_{0}$, the $k$-th superdiagonal $\mathbf{D}_{k}$ and the $k$-th subdiagonal $\mathbf{D}_{-k}$, respectively. ${ }^{1}$ Thus, for the general $k$-tridiagonal matrix we use notation $\mathbf{T}_{n}^{(k)}\left(\mathbf{D}_{-k}, \mathbf{D}_{0}, \mathbf{D}_{k}\right)$ or directly

$$
\mathbf{T}_{n}^{(k)}\left(\left\{b_{j}\right\}_{j=k+1}^{n},\left\{d_{j}\right\}_{j=1}^{n},\left\{a_{j}\right\}_{j=1}^{n-k}\right)
$$

but for the $k$-tridiagonal Toeplitz matrix we can write shortly $\mathbf{T}_{n}^{(k)}(b, d, a)$, since for diagonals of matrix (1) hold

$$
\left\{d_{j}=d\right\}_{j=1}^{n},\left\{a_{j}=a\right\}_{j=1}^{n-k}, \text { and }\left\{b_{j}=b\right\}_{j=k+1}^{n}
$$

Küçük, Düz [5] studied, recursive relations between the Chebyshev polynomials of the second kind (for more information, see [6]), which can be defined for $n>2$ by the recurrence relation

$$
U_{n}(x)=2 x U_{n-1}(x)-U_{n-2}(x)
$$

with initial values $U_{0}(x)=1$ and $U_{1}(x)=2 x$, and the permanents (the definition and many properties of permanents you can find in [7]) of a special type of matrix (1), namely $k$-tridiagonal symmetric Toeplitz matrix $\mathbf{T}_{n}^{(k)}(i, 2 x, i)$, where $i$ is the imaginary unit, i. e., the matrix with entries

$$
t_{j m}^{(k)}= \begin{cases}2 x, & j=m ; \\ i, & j=m \pm k \\ 0, & \text { otherwise }\end{cases}
$$

where $1 \leq j, m \leq n$.

To prove [5, Conjecture 8] first da Fonseca in [8] showed that the permanent of the matrix $\mathbf{T}_{n}^{(k)}(i, 2 x, i)$ is equal to the permanent of the matrix $\mathbf{T}_{n}^{(k)}(-1,2 x, 1)$, with respect to the fact, that the permanent of a square matrix equals the sum of the weights of all cycle-covers of its underlying directed graph. Then, he used a result on convertible matrices from his paper [10] (some generalizations can be found in [11]) to show that the permanent of matrix $\mathbf{T}_{n}^{(k)}(-1,2 x, 1)$ is equal to the determinant of the matrix $\mathbf{T}_{n}^{(k)}(1,2 x, 1)$. Thus, he derived that

$$
\operatorname{per} \mathbf{T}_{n}^{(k)}(i, 2 x, i)=\operatorname{det} \mathbf{T}_{n}^{(k)}(1,2 x, 1)
$$

Borowska et al. [12-14] dealt with determinants of some pentagonal and heptadiagonal symmetric Toeplitz matrices. Inter alia, they paid attention to the determinant of the following heptadiagonal matrix

\footnotetext{
${ }^{1}$ Here we use the notation for the numbering diagonals, which can be found, e.g., in [9].
} 


$$
\mathbf{A}_{n}=\left(\begin{array}{cccccccccc}
a & b & c & d & & & & & & \\
b & a & b & c & d & & & & & \\
c & b & a & b & c & d & & & & \\
d & c & b & a & b & c & d & & & \\
& d & c & b & a & b & c & d & & \\
& & \ddots & \ddots & \ddots & \ddots & \ddots & \ddots & \ddots & \\
& & & d & c & b & a & b & c & d \\
& & & & d & c & b & a & b & c \\
& & & & & d & c & b & a & b \\
& & & & & & d & c & b & a
\end{array}\right)_{n \times n}
$$

To find a recurrence relation for determinants of matrix $\mathbf{A}_{n}$ they introduced the following two auxiliary heptadiagonal matrices

$$
\mathbf{A}_{n}=\left(\begin{array}{cccccccccc}
a & b & c & d & & & & & & \\
b & a & b & c & d & & & & & \\
c & b & a & b & c & d & & & & \\
d & c & b & a & b & c & d & & & \\
& d & c & b & a & b & c & d & & \\
& & \ddots & \ddots & \ddots & \ddots & \ddots & \ddots & \ddots & \\
& & & d & c & b & a & b & c & d \\
& & & & d & c & b & a & b & c \\
& & & & & d & c & b & a & b \\
& & & & & & \mathbf{0} & d & c & b
\end{array}\right)_{n \times n}
$$

and

$$
\widehat{\mathbf{A}}_{n}=\left(\begin{array}{cccccccccc}
a & b & c & d & & & & & & \\
b & a & b & c & d & & & & & \\
c & b & a & b & c & d & & & & \\
d & c & b & a & b & c & d & & & \\
& d & c & b & a & b & c & d & & \\
& & \ddots & \ddots & \ddots & \ddots & \ddots & \ddots & \ddots & \\
& & & d & c & b & a & b & c & \mathbf{0} \\
& & & & d & c & b & a & b & d \\
& & & & & d & c & b & a & c \\
& & & & & & \mathbf{0} & d & c & a
\end{array}\right)_{n \times n}
$$

They denoted determinants of matrices $\mathbf{A}_{n}, \bar{A}_{n}$, and $\widehat{\mathbf{A}}_{n}$ by $W_{n}, \bar{W}_{n}$, and $\widehat{W}_{n}$, respectively, and derived the following system of linear recurrence relations (see formulae (4) and (5) in [14], where all the needed initial conditions can be found too) 


$$
\begin{aligned}
W_{n+7} & =a W_{n+6}+b d\left(b d-2 c^{2}\right) W_{n+3}+d^{2}\left(2 c^{3}-4 b c d+b^{2} c+a d^{2}\right) W_{n+2} \\
& +d^{3}\left(2 c^{2} d+b^{2} d-b c^{2}-d^{3}\right) W_{n+1}-b c d^{5} W_{n}-b \bar{W}_{n+6}+b c \bar{W}_{n+5} \\
& +d\left(2 a c-b^{2}\right) \bar{W}_{n+4}+b d^{2}(2 c-a) \bar{W}_{n+3}+d^{3}\left(2 b d-b^{2}-c^{2}\right) \bar{W}_{n+2} \\
& +c d^{4}(b-2 d) \bar{W}_{n+1}+b d^{6} \bar{W}_{n}-c^{2} \widehat{W}_{n+5}+d(b c-a d) \widehat{W}_{n+4}, \\
\bar{W}_{n+6} & =b W_{n+5}-b c d^{2} W_{n+2}+d^{3}\left(c^{2}-b d\right) W_{n+1}+c d^{5} W_{n}-c \bar{W}_{n+5} \\
& +b d \bar{W}_{n+4}+a d^{2} \bar{W}_{n+3}+b d^{3} \bar{W}_{n+2}-c d^{4} \bar{W}_{n+1}-d^{6} \bar{W}_{n}-c d \widehat{W}_{n+4}, \\
\widehat{W}_{n+2} & =a W_{n+1}-c^{2} W_{n}+2 c d \bar{W}_{n}-d^{2} \widehat{W}_{n}
\end{aligned}
$$

\section{Main result}

Küçük, Düz [5] formulated the following proposition (we have made a small technical textual modification, that does not change their assertion, to avoid copying the whole text above this proposition)

\section{Remark 1}

$$
\operatorname{per} \mathbf{T}_{n}^{(3)}(i, 2 x, i), \operatorname{per} \mathbf{T}_{n}^{(4)}(i, 2 x, i), \operatorname{per} \mathbf{T}_{n}^{(5)}(i, 2 x, i), \ldots
$$

cannot be written in terms of themselves, thus as a self-recurrence for every of these permanents individually.

Küçük, Düz formulated the proof of this Remark 1 for the case $\operatorname{per} \mathbf{T}_{n}^{(3)}(i, 2 x, i)$, but the idea of this proof is incorrect, what we show by proving that there is a selfrecurrence for $\operatorname{per} \mathbf{T}_{n}^{(3)}(i, 2 x, i)$.

For the simplification of notation, we will use for permanent of matrix $\mathbf{T}_{n}^{(3)}(i, 2 x, i)$ the following denotation

$$
p_{n}:=\operatorname{per} \mathbf{T}_{n}^{(3)}(i, 2 x, i)
$$

where $n$ is a positive integer.

Theorem 1 Let $n$ be any positive integer. The sequence $\left\{p_{n}\right\}$, defined by (6), satisfies the following recurrence relation for $n>8$

$$
p_{n}=2 x p_{n-1}-p_{n-2}+2 x p_{n-3}-4 x^{2} p_{n-4}+2 x p_{n-5}-p_{n-6}+2 x p_{n-7}-p_{n-8}
$$

with the initial values 


$$
\begin{aligned}
& p_{1}=2 x, p_{2}=4 x^{2}, p_{3}=8 x^{3}, \\
& p_{4}=4 x^{2}\left(4 x^{2}-1\right), p_{5}=2 x\left(4 x^{2}-1\right)^{2}, \\
& p_{6}=\left(4 x^{2}-1\right)^{3}, p_{7}=4 x\left(2 x^{2}-1\right)\left(4 x^{2}-1\right)^{2}, \\
& p_{8}=(4 x)^{2}\left(2 x^{2}-1\right)^{2}\left(4 x^{2}-1\right)
\end{aligned}
$$

Proof Combining identities (2) and (6) we get $p_{n}=\operatorname{det} \mathbf{T}_{n}^{(3)}(1,2 x, 1)$, but this determinant is a special case of the determinant of the heptadiagonal matrix $\mathbf{A}_{n}$ in (3), when we set $a=2 x, b=c=0$, and $d=1$. Similarly, we denote determinants of matrices $\overline{\mathbf{A}}_{n}$ and $\widehat{A}_{n}$ by $\bar{p}_{n}$ and $\widehat{p}_{n}$, respectively. Then, from (4) we get the following system of three homogeneous linear recurrences for sequences $\left\{p_{n}\right\},\left\{\bar{p}_{n}\right\}$ and $\left\{\widehat{p}_{n}\right\}$

$$
\begin{aligned}
& p_{n+6}=2 x p_{n+5}+2 x p_{n+1}-p_{n}-2 x \widehat{p}_{n+3}, \\
& \bar{p}_{n+6}=2 x \bar{p}_{n+3}-\bar{p}_{n}, \\
& \widehat{p}_{n+2}=2 x p_{n+1}-\widehat{p}_{n}
\end{aligned}
$$

Since we are only interested in the sequence $\left\{p_{n}\right\}$, we can omit the second recurrence from the previous system to take the following system of two linear recurrences for sequences $\left\{p_{n}\right\}$ and $\left\{\widehat{p}_{n}\right\}$

$$
\begin{aligned}
& p_{n+6}=2 x p_{n+5}+2 x p_{n+1}-p_{n}-2 x \widehat{p}_{n+3}, \\
& \widehat{p}_{n+2}=2 x p_{n+1}-\widehat{p}_{n}
\end{aligned}
$$

which can be easily reduced by substitution method to the self-recurrence (7) of the sequence $\left\{p_{n}\right\}$. Initial conditions (8) for $p_{i}, 1 \leq i \leq 7$, we easily get as special cases of (5) in [14] and the initial condition for $p_{8}$ we can compute from (4) in [14]. Thus, the proof is complete.

\section{Conclusions}

In this article, our main purpose was to show that the statement in [5, Remark 9] is incorrect. For this purpose, we have found the self-recurrence for the sequence of permanents of the 3-tridiagonal Toeplitz matrix $\mathbf{T}_{n}^{(3)}(i, 2 x, i)$. Our derivation was based on two substantial previous results. First, we used da Fonseca [8], in which the author showed that the permanent of matrix $\mathbf{T}_{n}^{(k)}(i, 2 x, i)$, studied by Küçük and Düz [5], is equal to the determinant of the matrix $\mathbf{T}_{n}^{(k)}(1,2 x, 1)$. Subsequently, we used Borowska and Łacińska [14], in which authors found the recurrence system for calculating determinants of the heptadiagonal Toeplitz matrices. 


\section{Acknowledgement}

The author is very grateful to the referees for their very helpful comments that helped to improve the quality of this paper, in particular by bringing our attention to a classical result of Egerváry and Szász [2].

The work has been supported by Specific Research Project of Faculty of Science, University of Hradec Králové, No. 2101, 2018.

\section{References}

[1] El-Mikkawy, M.E.A., \& Sogabe, T. (2010). A new family of $k$-Fibonacci numbers. Appl. Math. Comput., 215, 4456-4461.

[2] Egerváry, V.E., \& Szász, O. (1928). Einige Extremalprobleme im Bereiche der trigonometrischen Polynome. Math. Z., 27(1), 641-652.

[3] Losonczi, L. (1992). Eigenvalues and eigenvectors of some tridiagonal matrices. Acta Mathematica Hungarica, 60(3), 309-332.

[4] da Fonseca, C.M. \& Kowalenko, V. (2019). Eigenpairs of a family of tridiagonal matrices: three decades later. Acta Math. Hungar.

[5] Küçük, A.Z., \& Düz, M. (2017). Relationships between the permanents of a certain type of $k$-tridiagonal symmetric Toeplitz and the Chebyshev polynomials. Journal of Applied Mathematics and Computational Mechanics, 16, 75-86.

[6] Mason, J.C., \& Handscomb, D.C. (2003). Chebyshev Polynomials. Boca Raton, USA: Chapman and Hall/CRC Press Company.

[7] Minc, H. (1978). Permanents. Reading: Addison-Wesley.

[8] da Fonseca, C.M. (2018). On some conjectures regarding tridiagonal matrices. Journal of Applied Mathematics and Computational Mechanics, 17(4), 13-17.

[9] https://www.ibm.com/support/knowledgecenter/en/SSFHY8_6.1/reference/am5gr_cdst.html

[10] da Fonseca, C.M. (2011). An identity between the determinant and the permanent of Hessenberg type-matrices. Czechoslovak Mathematical Journal, 61(136), 917-921.

[11] da Cruz, H. F., Rodrigues, I. I., Serodio, R., Simoes, A., \& Velhinho, J. (2017). Convertible Subspaces of Hessenberg-Type Matrices. Mathematics, 5(4), Article number: 79.

[12] Borowska, A., Łacińska, L., \& Rychlewska, J. (2013). On determinant of certain pentadiagonal matrix. Journal of Applied Mathematics and Computational Mechanics, 12(3), 21-26.

[13] Borowska, A., Łacińska, L., \& Rychlewska, J. (2014). A system of linear recurrence equations for determinant of pentadiagonal matrix. Journal of Applied Mathematics and Computational Mechanics, 13(2), 5-12.

[14] Borowska, A., \& Łacińska, L. (2014). Recurrence form for determinant of a heptadiagonal symmetric Toeplitz matrix. Journal of Applied Mathematics and Computational Mechanics, 13, $19-26$. 